\title{
Response time of a normal-metal/superconductor hybrid system under a step-like pulse bias
}

\author{
Yanxia Xing, ${ }^{1}$ Qing-feng Sun, ${ }^{1, *}$ and Jian Wang ${ }^{2}$ \\ ${ }^{1}$ Beijing National Laboratory for Condensed Matter Physics and Institute of Physics, \\ Chinese Academy of Sciences, Beijing 100080, China \\ ${ }^{2}$ Department of Physics and the Center of Theoretical and Computational Physics, \\ The University of Hong Kong, Pokfulam Road, Hong Kong, China
}

(Received 29 November 2006; published 9 March 2007)

\begin{abstract}
The response of a quantum dot coupled with one normal lead and a superconductor lead driven by a step-like pulse bias $V_{L}$ is studied using the nonequilibrium Green's function method. In the linear pulse bias regime, the responses of the upward and downward biases are symmetric. In this regime, the turn-on time and turn-off time are much slower than those of the normal system due to the Andreev reflection. On the other hand, for the large pulse bias $V_{L}$, the instantaneous current exhibits oscillatory behaviors with the frequency $\hbar \Omega=q V_{L}$. The turn-on/off times are in (or shorter than) the scale of $1 / V_{L}$, so they are faster for the larger bias $V_{L}$. In addition, the responses for the upward and downward biases are asymmetric at large $V_{L}$. The turn-on time is larger than the turn-off time, but the relaxation time depends only on the coupling strength $\Gamma$ and it is much smaller than the turn-on/off times for the large bias $V_{L}$. [The turn-on/off time describes how fast a device can turn on/off a current, which is also named rise/fall time in M. Plihal et al., Phys. Rev. B 61, R13341 (2000), while the relaxation time was referred to how fast the device can go to a new steady state after a bias is abruptly switched on. It is also named saturation time in A. Schiller and S. Hershfield, Phys. Rev. B 62, R16271 (2000).]
\end{abstract}

DOI: 10.1103/PhysRevB.75.125308

PACS number(s): 73.23. - b, 74.25.Fy, 74.50.+r

\section{INTRODUCTION}

In the past two decades, nanoscopic physics has developed significantly and has become an active field of condensed-matter physics. The quantum transport property also has become one of the most interesting phenomena in nanoscopic physics because of the possibility of designing and fabricating artificial setups in the nanometer scale. Based on the transport physics in a nanoscopic system, a rich field for basic and applied research is opened. ${ }^{1}$ Furthermore, the time-dependent nanoscopic transport, in which the external time-dependent fields drive the electrons to tunnel through a nanoscopic system, has received increasing attention in recent years. The main feature of the transport in the nanometer scale is that the electron keeps the phase coherence when traversing through the device, while the external timedependent field affects the phase factor of the incident electron differently in different parts of the system. ${ }^{2}$ If the external time-dependent field is sinusoidal (e.g., microwave radiation), an electron can tunnel through the system by emitting or absorbing photons, giving rise to the photonassisted tunneling (PAT). Electron transport with PAT has been extensively investigated for various systems, such as single or two coupled quantum dot (QD), ${ }^{3-5}$ Kondo regime, ${ }^{6}$ hybrid system, ${ }^{7}$ and so on. For transient transport, one of the most interesting issues is how fast a device can turn on or turn off a current. With the development of the molecular devices, there is clearly a need to technologically provide a particular viable switching device. Indeed, some recent experimental and theoretical works have already begun to study the response of ac signals of the molecular devices. ${ }^{8}$ Consequently, step or pulsed ac signals are the simplest choice since they can provide a less ambiguous measure of time scales. For this reason, the pulsed field was studied in a variety of systems, including Kondo regime, ${ }^{9,10}$ a single $\mathrm{QD},{ }^{11}$ or nanostructure. ${ }^{12,13}$
So far, the study of the response of a pulsed bias is only focused on normal nanostructures. Since the interplay between nanoscopic physics and the physics of superconductivity has made the hybrid structure a very fruitful research field, ${ }^{14}$ it will be interesting to study the dynamic response of a hybrid structure with a superconductor lead, where the Andreev reflection is present near the normal-superconductor $(\mathrm{N}-\mathrm{S})$ interface. Indeed, there are many interesting phenomena in the N-S hybrid systems. First of all, because there exists an energy gap $\Delta$ in the superconductor, an incident electron from the normal side with energy $\epsilon$ inside the gap $\Delta$ cannot tunnel into the superconductor. But the tunneling can occur via a two-particle process, in which the incident electron is reflected as a hole with the energy $-\epsilon$. At the same time, a Cooper pair is created in the superconductor region. This is the Andreev reflection. ${ }^{15}$ Second, for the superconductor-normal region-superconductor ( $\mathrm{S}-\mathrm{N}-\mathrm{S})$ system, Andreev bound states form in the normal region due to the Andreev reflections at N-S interfaces. ${ }^{16}$ These bound states exist in pairs, and a Josephson supercurrent can flow through the S-N-S system, which is carried by the Andreev bound state. ${ }^{17}$ Third, when the S-N-S device is under an external dc bias $V$, an ac with frequency $\omega=2|e| V$ appears. The time-average current versus bias $V$ exhibits the subharmonic gap structure when $\mathrm{eV}<2 \Delta .{ }^{18}$

In this paper, we explore the effect of Andreev reflection on the ac response of a hybrid system. Specifically, we investigate the ac response of a QD with a single level $\epsilon_{0}$ connected by a normal and a superconductor lead (N-QD-S). For simplicity, we consider a large QD so that the intradot electron-electron $(e-e)$ is weak and can be neglected. ${ }^{19}$ The transient transport is driven by a pulsed bias potential $W(t)$. For simplicity, the ac pulsed bias is only added in the left lead, and we set $W_{R}(t)=0$. We consider two different pulsed biases: (i) upward pulse with $W_{L}(t)=0$ for $t<0$ and $W_{L}(t)$ 
$=V_{L}$ otherwise; (ii) downward pulse with $W_{L}(t)=V_{L}$ when $t$ $<0$ and $W_{L}(t)=0$ otherwise. For normal structures, Wingreen et al. presented a general formula for the current driven by the time-dependent external fields by using the nonequilibrium Green's function method. ${ }^{2,11}$ With this general formula, the time-dependent current driven by the ac pulse can be calculated. For hybrid structures, the system is in a steady state at $t<0$ and the current is time independent. At $t=0$, the bias is abruptly turned on for the upward pulse case or turned off for the downward pulse case. After that, the system begins to relax and the Andreev reflection plays an important role in the relaxation process. Finally, the system enters into a new steady state. We find that the relaxation time depends on the coupling strength and is slower in the N-QD-S system (named hybrid system hereafter) than in the N-QD-N system (named normal system hereafter). In the linear bias regime, the rising and falling processes are symmetric so that the turn-on time is the same as the turn-off time. In this regime, the Andreev reflection is important. As a result, the instantaneous current shows a clear increase (decrease) before reaching the new steady state for the downward (upward) pulse. For the large bias case, the time-dependent current oscillates with the frequency $\omega=q V_{L}$. In this regime, the upward and downward processes are asymmetric and the turn-on time is much larger than the turn-off time. In this nonlinear regime, the Andreev process is negligible and the current in the hybrid system is close to that of the normal system.

The rest of this paper is organized as follows: In Sec. II, the theoretical formula for calculating the time-dependent current in the N-QD-S system is presented. To understand the numerical results, the current away from the current at $t=0$ is expanded to first order in the external bias. In Sec. III, we show the numerical results along with some discussions. Finally, the brief summary is given in Sec. IV.

\section{THEORETICAL FORMULA}

Consider a hybrid system that consists of a QD coupled to a normal metal lead and a superconductor lead with the external time-dependent bias potential $W_{L}(t)$ that is added only on the left normal lead. The Hamiltonian of the system is written as follows:

$$
H=H_{L}+H_{R}+H_{D}+H_{T},
$$

where $H_{L}$ and $H_{R}$ describe the left normal lead and the right superconductor lead, respectively. $H_{D}$ is the Hamiltonian of the isolated central QD, and $H_{T}$ couples the left and right leads to the QD. They can be written in the following forms ${ }^{20,21}$

$$
\begin{gathered}
H_{L}=\sum_{k \sigma}\left(\epsilon_{L, k}+W_{L}(t)\right) C_{L, k \sigma}^{\dagger} C_{L, k \sigma}, \\
H_{R}=\sum_{k \sigma} \epsilon_{R, k} C_{R, k \sigma}^{\dagger} C_{R, k \sigma}+\sum_{k}\left[\Delta C_{R, k \downarrow} C_{R,-k \uparrow}+\Delta C_{R,-k \uparrow}^{\dagger} C_{R, k \downarrow}^{\dagger}\right], \\
H_{D}=\sum_{\sigma} \epsilon_{0} d_{\sigma}^{\dagger} d_{\sigma},
\end{gathered}
$$

$$
H_{T}=\sum_{\sigma, k, \alpha} t_{k, \alpha} C_{\alpha, k \sigma}^{\dagger} d_{\sigma}+\text { H.c. },
$$

where $\alpha=L, R$. The operators $d_{\sigma}$ and $C_{\alpha, k \sigma}$ destroy an electron with spin $\sigma$ in the QD and in the left or right lead, respectively. For simplicity, we only consider a single level in the QD and neglect the intradot electron-electron Coulomb interaction. Under the adiabatic approximation, the time-dependent bias potential can be included in the singleelectron energy $\epsilon_{L, k}(t)$. We separate $\epsilon_{L, k}(t)$ into two parts: $\epsilon_{L, k}$ and $W_{L}(t)$, where $\epsilon_{L, k}$ is the time-independent single-electron energy and $W_{L}(t)$ is a time-dependent part from the external time-dependent bias potential. In this paper, $W_{L}(t)$ is the step-like pulse with two different forms: (i) upward pulse with $W_{L}(t)=0$ when $t<0$ and $W_{L}(t)=V_{L}$ otherwise; (ii) downward pulse with $W_{L}(t)=V_{L}$ when $t<0$ and $W_{L}(t)=0$ otherwise. These two types of pulse describe the system abruptly turned on or turned off at time $t=0 . \Delta$ in the Hamiltonian $H_{R}$ is the superconducting energy gap. We assume that $\Delta$ is a real parameter by selecting a special phase of the superconductor lead in our calculation. ${ }^{22}$ Due to the existence of the superconducting lead, it is convenient to introduce the Nambu representation. ${ }^{23}$ In the Nambu representation, the Fermi energy of the left normal lead is set at the superconducting condensate. For the spin-down electron, the energy is negative and is viewed as the hole. So, the Hamiltonian in Eqs. (2) can be rewritten in the following matrix forms:

$$
\begin{gathered}
H_{L}=\sum_{k} \Psi_{L, k}^{\dagger}\left(\begin{array}{cc}
\epsilon_{L, k}+W_{L}(t) & 0 \\
0 & -\epsilon_{L,-k}-W_{L}(t)
\end{array}\right) \Psi_{L, k}, \\
H_{R}=\sum_{k} \Psi_{R, k}^{\dagger}\left(\begin{array}{cc}
\epsilon_{R, k} & \Delta \\
\Delta & -\epsilon_{R,-k}
\end{array}\right) \Psi_{R, k}, \\
H_{D}=\Phi^{\dagger}\left(\begin{array}{cc}
\epsilon_{0} & 0 \\
0 & -\epsilon_{0}
\end{array}\right) \Phi, \\
H_{T}=\sum_{k, \alpha} \Psi_{k, \alpha}^{\dagger}\left(\begin{array}{cc}
t_{k, \alpha, \uparrow} & 0 \\
0 & -t_{k, \alpha, \uparrow}^{*}
\end{array}\right) \Phi+\text { H.c. },
\end{gathered}
$$

where

$$
\Psi_{\alpha, k}=\left(\begin{array}{c}
C_{\alpha, k \uparrow} \\
C_{\alpha,-k \downarrow}^{\dagger}
\end{array}\right), \quad \Phi=\left(\begin{array}{c}
d_{\uparrow} \\
d_{\downarrow}^{\dagger}
\end{array}\right) .
$$

The current from the left lead to the QD can be calculated from the evolution of the number operator of the electrons in the left lead, $\left.N_{L, \uparrow(\downarrow)}=\Sigma_{k} C_{\alpha, k \uparrow(\downarrow)}^{\dagger} C_{\alpha, k \uparrow(\downarrow)}\right)^{2,11,21}$ Using the Keldysh equation and the theorem of analytic continuation, the current through the left normal metal lead is expressed $\mathrm{as}^{2,21,24}$

$$
\begin{aligned}
J_{L}(t)= & -2 q \operatorname{Re} \int_{-\infty}^{t} d t^{\prime}\left\{\left[G^{r}\left(t, t^{\prime}\right) \Sigma_{L}^{<}\left(t^{\prime}, t\right)\right.\right. \\
& \left.+G^{<}\left(t, t^{\prime}\right) \Sigma_{L}^{a}\left(t^{\prime}, t\right)\right]_{11}-\left[G^{r}\left(t, t^{\prime}\right) \Sigma_{L}^{<}\left(t^{\prime}, t\right)\right. \\
& \left.\left.+G^{<}\left(t, t^{\prime}\right) \Sigma_{L}^{a}\left(t^{\prime}, t\right)\right]_{22}\right\} .
\end{aligned}
$$


Here, the Green's function $G^{r /<}$ and the self-energy $\Sigma^{</ a}$ are all two-dimensional matrices in the Nambu representation. Since the spin up and spin down are symmetric in the Hamiltonian, the current contributed by the electrons with spin up is the same as the current by the spin down electrons. Consequently, the current is given by

$$
\begin{aligned}
J_{L}(t)= & -4 q \operatorname{Re} \int_{-\infty}^{t} d t^{\prime}\left[G^{r}\left(t, t^{\prime}\right) \Sigma_{L}^{<}\left(t^{\prime}, t\right)\right. \\
& \left.+G^{<}\left(t, t^{\prime}\right) \Sigma_{L}^{a}\left(t^{\prime}, t\right)\right]_{11} .
\end{aligned}
$$

Because of $\Sigma_{L}^{a}\left(t, t^{\prime}\right)=\left[\Sigma_{L}^{r}\left(t, t^{\prime}\right)\right]^{\dagger}=\left(i \Gamma_{L} / 2\right) \delta\left(t-t^{\prime}\right) \mathbf{I}$ (see Appendix), where $\mathbf{I}$ is the 2 unit matrix, only $G^{<}(t, t)$ instead of $G^{<}\left(t, t^{\prime}\right)$ is needed in Eq. (6). By using the Keldysh equation $G^{<}=G^{r} \Sigma<G^{a}$ with the self-energies obtained in the Appendix, the Green's function $G^{<}(t, t)$ can be solved by

$$
\begin{aligned}
G^{<}(t, t)= & \sum_{\alpha} \int d t_{1} \int d t_{2} G^{r}\left(t, t_{1}\right) \Sigma_{\alpha}^{<}\left(t_{1}, t_{2}\right) G^{a}\left(t_{2}, t\right) \\
= & i \int \frac{d \omega}{2 \pi} f(\omega) G^{r}(\omega) \bar{\Gamma}_{R}(\omega) G^{a}(\omega) \\
& +i \sum_{\sigma} \int \frac{d \omega}{2 \pi} f(\omega) A_{L, \sigma}(\omega, t) s_{\sigma} \Gamma_{L}(\omega) A_{L, \sigma}^{+}(\omega, t),
\end{aligned}
$$

where $\sigma= \pm 1$ denotes the spin up $\uparrow$ and spin down $\downarrow$,

$$
\bar{\Gamma}_{R}(\omega)=\theta(\omega-\Delta) \frac{\Gamma_{R}}{\sqrt{\omega^{2}-\Delta^{2}}}\left(\begin{array}{cc}
|\omega| & \Delta \\
\Delta & |\omega|
\end{array}\right),
$$

and

$$
\begin{aligned}
s_{\uparrow}= & \left(\begin{array}{ll}
1 & 0 \\
0 & 0
\end{array}\right), \quad s_{\downarrow}=\left(\begin{array}{ll}
0 & 0 \\
0 & 1
\end{array}\right), \\
A_{L, \sigma}(\omega, t)= & \int_{-\infty}^{t} d t_{1} G^{r}\left(t, t_{1}\right) \exp \left[i \omega\left(t-t_{1}\right)\right. \\
& \left.+i \sigma \int_{t_{1}}^{t} d t_{2} W_{L}\left(t_{2}\right)\right]
\end{aligned}
$$

The Green's functions $G^{r / a}(\omega)$ in Eq. (7) are the Fourier transformation of $G^{r / a}\left(t, t^{\prime}\right) \quad$ with $\quad G^{r / a}(\omega)=\int d(t$ $\left.-t^{\prime}\right) e^{i \omega\left(t-t^{\prime}\right)} G^{r / a}\left(t, t^{\prime}\right)$. Notice that in the present system, the retarded and advanced Green's functions $G^{r / a}\left(t, t^{\prime}\right)$ are still the function of the time difference $t-t^{\prime}$, although there exists the time-dependent bias $W_{L}(t)$, since $G^{r}(\omega)$ can be obtained from the Dyson equation

$$
\begin{aligned}
G^{r}(\omega) & =\left[\omega-H_{d o t}-\Sigma_{L}^{r}-\Sigma_{R}^{r}\right]^{-1} \\
& =\frac{1}{\operatorname{Det}}\left(\begin{array}{cc}
B_{11} & i \nu \Gamma_{R} \beta^{\prime} / 2 \\
i \nu \Gamma_{R} \beta^{\prime} / 2 & B_{22}
\end{array}\right),
\end{aligned}
$$

where $\quad B_{11}=\omega+\epsilon_{0}+i \Gamma_{L} / 2+i \nu \Gamma_{R} \beta / 2, \quad B_{22}=\omega-\epsilon_{0}+i \Gamma_{L} / 2$ $+i \nu \Gamma_{R} \beta / 2, \quad \beta=\Delta / \sqrt{\omega^{2}-\Delta^{2}}, \quad \beta^{\prime}=\omega / \sqrt{\omega^{2}-\Delta^{2}}, \quad$ Det $=B_{11} B_{22}$ $+\left(\Gamma_{R} \beta^{\prime}\right)^{2} / 4$, and $\nu=1$ for $\omega>-\Delta$ and $\nu=-1$ otherwise. In the above derivation, the wideband limit has been used and
$\Gamma_{\alpha}$ are assumed independent of $\omega \cdot{ }^{25}$ It is also worth mentioning that the Green's function $G^{r / a}(\omega)$ is not affected by the time-dependent bias potential $W_{L}(t)$.

Substituting $G^{<}(t, t)$ [in Eq. (7)] and the self-energies $\Sigma^{<l a}\left(t^{\prime}, t\right)$ (in the Appendix) into Eq. (6), the time-dependent current $J_{L}(t)$ is obtained straightforwardly. Similar to the work in the normal system by Jauho et al., ${ }^{2}$ the current $J_{L}(t)$ can also be split into two terms $J_{L}^{\text {in }}(t)$ and $J_{L}^{\text {out }}(t)$ :

$$
\begin{gathered}
J_{L}^{\text {in }}(t)=4 q \int \frac{d \omega}{2 \pi} f(\omega) \operatorname{Im}\left\{\Gamma_{L}\left[A_{L \uparrow}(\omega, t)\right]_{11}\right\}, \\
J_{L}^{\text {out }}(t)=-2 q \int \frac{d \omega}{2 \pi} f(\omega) \operatorname{Re}\left\{\Gamma _ { L } \left[G^{r}(\omega) \bar{\Gamma}_{R}(\omega) G^{a}(\omega)\right.\right. \\
\left.\left.+\sum_{\sigma} A_{L \sigma}(\omega, t) s_{\sigma} \Gamma_{L} A_{L \sigma}^{+}(\omega, t)\right]_{11}\right\},
\end{gathered}
$$

and $J_{L}(t)=J_{L}^{\text {in }}(t)-J_{L}^{\text {out }}(t)$. Here, the current $J_{L}^{\text {in }}(t)$ is contributed by the electrons tunneling from the left lead to the empty QD, and the current $J_{L}^{\text {out }}(t)$ describes the electrons tunneling from the QD to the empty left lead, so they have opposite signs. $^{2}$

The above formulations [Eqs. (9)-(11)] for calculating the current are valid for any time-dependent bias $W_{L}(t)$. In the following, two special cases for upward and downward pulses $W_{L}(t)$ are substituted into these formulations to obtain $A_{L \sigma}(\epsilon, t)\left[\right.$ Eq. (9)] and then the currents $J_{L}^{i n}(t)$ and $J_{L}^{\text {out }}(t)$ [Eqs. (11)].

For the downward pulse with $W_{L}(t<0)=V_{L}$ and $W_{L}(t$ $>0)=0, A_{L \uparrow}(\epsilon, t)$ is found to be

$$
\begin{gathered}
A_{L D, \uparrow}(\omega, t<0)=G^{r}\left(\omega+V_{L}\right), \\
A_{L D, \uparrow}(\omega, t>0)=G^{r}(\omega)+\int \frac{d E}{2 \pi i} e^{-i(E-\omega) t} G^{r}(E) \\
\\
\times\left[\frac{1}{E-\omega-V_{L}-i 0^{+}}-\frac{1}{E-\omega-i 0^{+}}\right] .
\end{gathered}
$$

For the upward pulse with $W_{L}(t<0)=0$ and $W_{L}(t>0)$ $=V_{L}, A_{L \uparrow}(\epsilon, t)$ are

$$
\begin{gathered}
A_{L U, \uparrow}(\omega, t<0)=G^{r}(\omega), \\
A_{L U, \uparrow}(\omega, t>0)=G^{r}\left(\omega+V_{L}\right)-\int \frac{d E}{2 \pi i} e^{-i\left(E-\omega-V_{L}\right) t} G^{r}(E) \\
\times\left[\frac{1}{E-\omega-V_{L}-i 0^{+}}-\frac{1}{E-\omega-i 0^{+}}\right]
\end{gathered}
$$

Here, $A_{L \sigma}(\epsilon, t)$ for the downward and upward pulse biases have been labeled by $A_{L D, \sigma}(\epsilon, t)$ and $A_{L U, \sigma}(\epsilon, t)$, respectively. For $t<0$, the system is in the steady state, so $A_{L D, \sigma}(\epsilon, t)$ and $A_{L U, \sigma}(\epsilon, t)$ are independent of time $t$. On the other hand, for $t>0$, they are obviously dependent on time $t$. For the purpose of numerical calculation, we rewrite $A_{L D / U, \sigma}(\epsilon, t)$ for $t>0$ in the following form by using the residue theorem: 


$$
\begin{aligned}
A_{L D, \uparrow}(\omega, t>0)= & G^{r}(\omega)+e^{-i V_{L} t} \int_{t}^{\infty} d \tau e^{i\left(\omega+V_{L}\right) \tau} G^{r}(\tau) \\
& -\int_{t}^{\infty} d \tau e^{i \omega \tau} G^{r}(\tau), \\
A_{L U, \uparrow}(\omega, t>0)= & G^{r}\left(\omega+V_{L}\right)+e^{i V_{L} t} \int_{t}^{\infty} d \tau e^{i \omega \tau} G^{r}(\tau) \\
& -\int_{t}^{\infty} d \tau e^{i\left(\omega+V_{L}\right) \tau} G^{r}(\tau) .
\end{aligned}
$$

The expressions of $A_{L, \downarrow}(\omega, t)$ are similar to that of $A_{L \uparrow}(\omega, t)$ and can be obtained from Eq. (14) by changing $V_{L}$ to $-V_{L}$. After solving $G^{r}(\omega)$ and $A_{L \sigma}(\omega, t)$, the currents $J_{L}^{\text {in }}(t)$ and $J_{L}^{\text {out }}(t)$ [Eq. (11)] can be calculated straightforwardly. In the limits $t \leqslant 0$ and $t \rightarrow \infty$, the system is in the steady state. $A_{L D, \sigma}(\omega, t)$ and $A_{L U, \sigma}(\omega, t)$ in Eq. (14) are then reduced to the value of the steady state in these two limits, and so is the current $J_{L}(t)$. For example, for the downward pulse, $A_{L D, \sigma}(\omega, t)=G^{r}\left(\omega+\sigma V_{L}\right)$ for $t \rightarrow 0$ and $A_{L D, \sigma}(\omega, t)=G^{r}(\omega)$ when $t \rightarrow \infty$. Furthermore, the current $J_{L}(t)$ is reduced to that of the steady-state case with dc bias $V_{L}$ when $t \leqslant 0$ and is zero when $t \rightarrow \infty$. On the other hand, for the upward pulse, the current $J_{L}(t)$ is zero when $t \leqslant 0$ and is the same with the steady-state current with the dc bias $V_{L}$ in $t \rightarrow \infty$ limit.

In the small pulse bias $V_{L}$ limits, we can expand $A_{L \sigma}(\omega, t>0)$ to the first order of $V_{L}$ as $A_{L \sigma}(\omega, t>0)$ $=A_{L \sigma}(\omega, t=0)+A_{L \sigma}^{1}(\omega, t>0) V_{L} . \quad A_{L \sigma}^{1}(\omega, t>0) \quad$ can be expressed as

$$
\begin{aligned}
& A_{L D, \sigma}^{1}(\omega, t>0)=-i \sigma t \int_{t}^{\infty} d \tau e^{i \omega \tau} G^{r}(\tau)-\sigma \int_{0}^{t} d \tau i \tau e^{i \omega \tau} G^{r}(\tau), \\
& A_{L U, \sigma}^{1}(\omega, t>0)=i \sigma t \int_{t}^{\infty} d \tau e^{i \omega \tau} G^{r}(\tau)+\sigma \int_{0}^{t} d \tau i \tau e^{i \omega \tau} G^{r}(\tau) .
\end{aligned}
$$

From Eq. (15), we can see that $A_{L D, \sigma}^{1}(\omega, t)=-A_{L U, \sigma}^{1}(\omega, t)$. This means that the upward pulse and the downward pulse induce the same relaxation process in the small pulse bias $V_{L}$ limits, except that the currents deduced from them are relaxed in the opposite direction. Finally, the currents $J_{L}^{i n}(t)$ and $J_{L}^{\text {out }}(t)$ in small $V_{L}$ limits can also be expanded as $J_{L}^{\text {in/out }}(t)$ $=J_{L}^{\text {in/out }}(0)+X^{\text {in/out }}(t) V_{L}$. Here, $X^{\text {in/out }}(t)$ is the first-order expansion coefficient with respect to $V_{L}$, and $X^{\text {in/out }}(t)$ is expressed as

$$
\begin{gathered}
X^{\text {in }}(t)=4 q \int \frac{d \omega}{2 \pi} \operatorname{Im} f(\omega) \Gamma_{L}\left\{A_{L, \uparrow}^{1}(\omega, t)\right\}_{11}, \\
X^{\text {out }}(t)=-2 q \int \frac{d \omega}{2 \pi} \operatorname{Re} f(\omega) \Gamma_{L} \sum_{\sigma}\left\{A_{L, \sigma}^{1}(\omega, t) s_{\sigma} \Gamma_{L} G^{a}(\omega)\right. \\
\left.+G^{r}(\omega) s_{\sigma} \Gamma_{L}\left[A_{L, \sigma}^{1}(\omega, t)\right]^{\dagger}\right\}_{11} .
\end{gathered}
$$

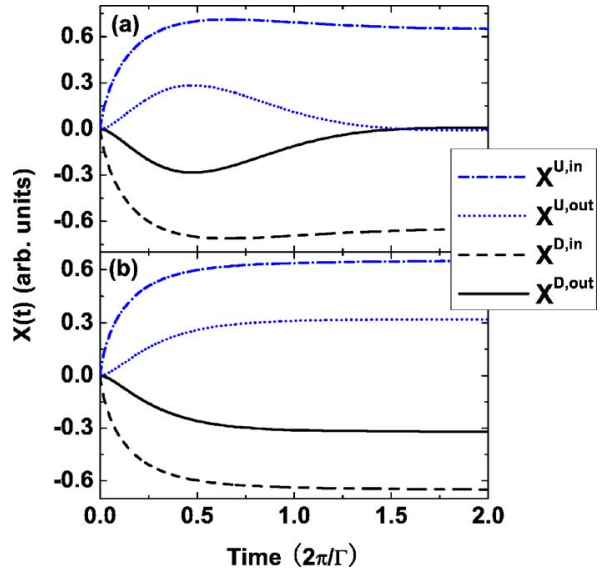

FIG. 1. (Color online) The first-order expansion coefficient $X(t)$ of the current $J_{L}(t)-J_{L}(0)$ vs time $t$ for the downward and upward pulse bias cases in the hybrid N-QD-S system (a) and the normal N-QD-N system (b). The parameters are $\Gamma=1, \delta \Gamma=0, \Delta=15$, and $\epsilon_{0}=0$.

\section{NUMERICAL RESULTS AND DISCUSSIONS}

In the numerical calculation, we set temperature to zero. In fact, a finite temperature only makes the current curve smoother and does not affect the main features. We focus on the weak coupling case with $\Gamma_{L / R} \ll \Delta$ and set $\Gamma=\Gamma_{L}+\Gamma_{R}=1$ as an energy unit. The energy gap of the superconductor is $\Delta=15$. The energy level $\epsilon_{0}$ in the central region is assumed to be zero, which is the same as the right Fermi level. Because the system is in the steady state and the current is time independent at $t \leqslant 0$, we only plot the current $J_{L}(t)$ and the related quantities for $t \geqslant 0$ in the following discussion.

First of all, we study the small pulse bias $V_{L}$ limit, in which the instantaneous current $J_{L}(t)$ can be expanded as $J_{L}(t)=J_{L}(0)+X(t) V_{L}$, and we also take the symmetric barriers, i.e., $\delta \Gamma=\Gamma_{L}-\Gamma_{R}=0$. The first-order expansion parameters $X^{U / D, \text { in/out }}(t)$ of the currents $J_{L}^{\text {in }}(t)$ and $J_{L}^{\text {out }}(t)$ versus time $t$ are plotted in Fig. 1. Here, the indices $U$ and $D$ denote the upward and downward pulses, respectively. For comparison, we also show the corresponding parameters $X^{U / D \text {,in/out }}(t)$ for the normal system in Fig. 1(b). In Fig. 1, we can see that the expanding parameters $X(t)$ for the upward and downward pulses are symmetric, i.e., $X^{U \text {,in/out }}(t)=-X^{D \text {,in/out }}(t)$. It means that in the small $V_{L}$ limit (i.e., the linear regime), the current was turned off or turned on by the downward or upward pulses in exactly the same manner with the same time scale for both normal system and hybrid system. In other words, the case of the downward pulse is the reversal process of the upward pulse. So, in the following, we use the upward pulse as an example in the linear region.

At time $t \leqslant 0$, the driving bias is zero for the upward case. The system is in equilibrium state, so the current $J_{L}^{U}$ is zero, and $J_{L}^{U, \text { in }}$ and $J_{L}^{U, \text { out }}$ cancel each other. At $t=0$, the bias is abruptly switched on. At $t>0$, the bias $W_{L}(t)$ is kept at $V_{L}$ all along. The electrons with the energy in the bias window begin to traverse through QD. As time $t$ increases, $J_{L}^{U, \text { in }}$ and $J_{L}^{U, \text { out }}$ deviate from the initial value $(t=0)$. A net current gradually increases and the device is gradually turned on. As 
a result, for time $t$ from 0 to about $0.5(2 \pi / \Gamma), X^{U, i n}(t)$ and $X^{U, \text { out }}(t)$ gradually increase (see Fig. 1). This increasing process is almost the same for the normal system and the hybrid system. For the normal N-QD-N device, the relaxation process completes near time $t=0.5(2 \pi / \Gamma)$, and $X^{U, \text { out }}(t)$ is half of $X^{U, i n}(t)$ at large time. On the other hand, for the hybrid $\mathrm{N}$-QD-S device, the behavior of $X^{U, i n}(t)$ is approximatively the same as that of N-QD-N at large time, but $X^{U, \text { out }}(t)$ begins to decrease when $t>0.5(2 \pi / \Gamma)$, and it goes to zero at the end of the relaxation process. So the current $J_{L}^{U}(t=\infty)$ for the $\mathrm{N}-\mathrm{QD}-\mathrm{S}$ device is twice as large as that of the N-QD-N device. We interpret these properties as follows. For the normal system, the fact that $X^{i n}(t=\infty)$ is twice of $X^{\text {out }}(t=\infty)$ is because $X^{\text {in }}(t=\infty)$ and $X^{\text {out }}(t=\infty)$ are, respectively, contributed by the electrons tunneling from the left lead into the empty QD and from the QD into the empty left lead with the electronic energy $\omega$ between 0 and $V_{L}$. In this energy range the distribution of the left lead is $f_{L}(\omega)=1$, but the distribution in the QD is $\left[f_{L}(\omega)+f_{R}(\omega)\right] / 2=1 / 2$ for $t=\infty$. While for the hybrid N-QD-S system, after the bias is turned on, the Andreev reflection begins to play a role. For $J_{L}^{U, \text { in }}$, there is not much difference between the normal and hybrid systems since the electrons always tunnel from the left lead into the QD in both systems. But for $J_{L}^{U, \text { out }}$, instead of reflecting electrons from the QD into the left lead in the normal system, the Andreev process reflects back the hole out of the QD, which decreases $J_{L}^{U, \text { out }}$. Note that $T_{A}$ can be expressed as ${ }^{21}$

$$
T_{A}=\frac{\Gamma^{4}}{64 \omega^{4}+\left(\Gamma^{2}+\delta \Gamma^{2}\right)^{2}} .
$$

In the small bias limit $(\omega \approx 0)$ and $\delta \Gamma=0$, nearly all of the incoming electrons participate in the Andreev reflection. Because of this, $J_{L}^{U, \text { out }}(t=\infty)$ goes back to the initial $(t=0)$ value. So, $X^{\text {out }}(t)$ decreases to zero at $t=\infty$.

Next, we study the case of large pulse $V_{L}$. Figures 2(c) and 2(d) depict the currents $J_{L}^{\text {out }}$ and $J_{L}^{\text {in }}$ versus time $t$ for the large pulse strength $V_{L}=10$. For comparison, $J_{L}^{\text {out }}$ and $J_{L}^{\text {in }}$ for the small pulse strength $V_{L}=0.1$ are also plotted in Figs. 2(a) and 2(b). The currents $J_{L}^{\text {out }}$ and $J_{L}^{\text {in }}$ in the large bias case have the following characteristics: (i) In the small bias limit, the relaxation processes of upward and downward are symmetric. However, in the large pulsed bias $V_{L}$ case, they are asymmetric [see Figs. 2(c) and 2(d)]. For the larger pulse bias $V_{L}$, the asymmetry is stronger. (ii) For the large bias case, $J_{L}^{U, \text { in }}$ for the upward pulse oscillates with the frequency $\hbar \Omega=q V_{L}$, which can be clearly seen in Figs. 2(c) and 2(d) for $V_{L}=10$. At $V_{L}=0.1$ the oscillation disappears because $\hbar \Omega=q V_{L}$ is too small to oscillate before the system is completely relaxed. (iii) $J_{L}^{U \text {,out }}\left(J_{L}^{D \text {,out }}\right)$ of the hybrid system increases (decreases) at first and then decreases (increases), and it reaches a maximum (minimum) before the current relaxed completely. This is different from the normal system, in which the currents $J_{L}^{U, \text { out }}$ and $J_{L}^{D, \text { out }}$ are monotonously relaxed into the steady state. (iv) The decreasing (increasing) process of the current $J_{L}^{U, \text { out }}\left(J_{L}^{D, \text { out }}\right)$ in the large bias case is much weaker than that of the small bias case [see Figs. 2(b) and 2(d)]. Because for the large pulse, the energy of the incident electrons $\omega$ is

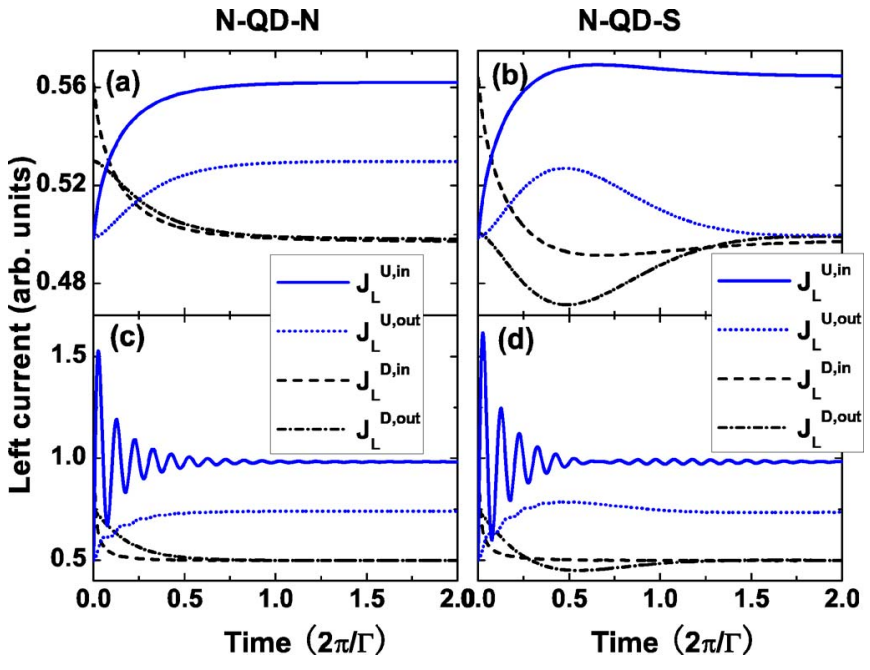

FIG. 2. (Color online) The currents $J_{L}^{U / D, \text { in }}$ and $J_{L}^{U / D \text {,out }}$ vs time $t$ for the small pulse bias $V_{L}=0.1$ [(a) and (b)] and the large pulse bias $V_{L}=10[(\mathrm{c})$ and (d)] in upward and downward pulse cases. (a) and (c) are for the N-QD-N system and (b) and (d) are for the N-QD-S system. The other parameters are the same as those in Fig. 1.

large, then $T_{A} \ll 1$ from Eq. (17) and the Andreev reflection is weak. So, most of the incident electrons participate in the normal reflection. Consequently, $J_{L}^{\text {out }}$ is humped up (or down) slightly.

Since the currents $J_{L}^{\text {out }}$ and $J_{L}^{\text {in }}$ cannot be observed independently, in the following we study the total current $J_{L}(t)$ $\left(J_{L}=J_{L}^{i n}-J_{L}^{\text {out }}\right)$ which can be measured in the experiment. Figure 3 shows the current $J_{L}^{U, D}$ driven by the upward and downward pulses versus time $t$ for the different pulse strengths $V_{L}$. Here, the current responses to the upward and downward pulses are symmetric at small linear bias $V_{L}$ (see inset of Fig. 3), but are asymmetric at the large bias $V_{L}$ (see the main part of Fig. 3). At the large $V_{L}, J_{L}^{U}$ oscillates with the frequency $\hbar \Omega=V_{L}$. On the other hand, $J_{L}^{D}$ always changes slowly regardless of the large and small $V_{L}$.

Now, we focus the turn-on/off time (or rise/fall time ${ }^{9}$ ) and the relaxation time (or saturation time ${ }^{10}$ ). The former describes how fast a device can turn on/off a current, which is necessary to provide a particular viable switching device, and the latter was referred to how fast the device can go to a new steady state after a bias is abruptly switched on. For the small bias $V_{L}$, the turn-on time, the turn-off time, and the relaxation time are almost the same regardless of the normal and hybrid systems. However, these (turn on/off or relaxation) times for the normal N-QD-N device are much shorter than that of the hybrid N-QD-S device. For the normal device, it has been well turned on or off at $t=0.2(2 \pi / \Gamma)$. But for the hybrid device, the system is turned on or off until $t$ $=1.0(2 \pi / \Gamma)$. On the other hand, for the large bias, the current $J_{L}(t)$ of the hybrid system has the same character as that of the normal system, so do the turn-on/off time and the relaxation time. Note that these three time scales are not equal now. The turn-on time is the fastest, even faster than the scale $1 / V_{L}(2 \pi / \Gamma)$. The turn-off time is in the scale $1 / V_{L}(2 \pi / \Gamma)$, which is longer than the turn-on time. ${ }^{9}$ The relaxation is $\sim 0.5(2 \pi / \Gamma)$, which is the longest and only de- 


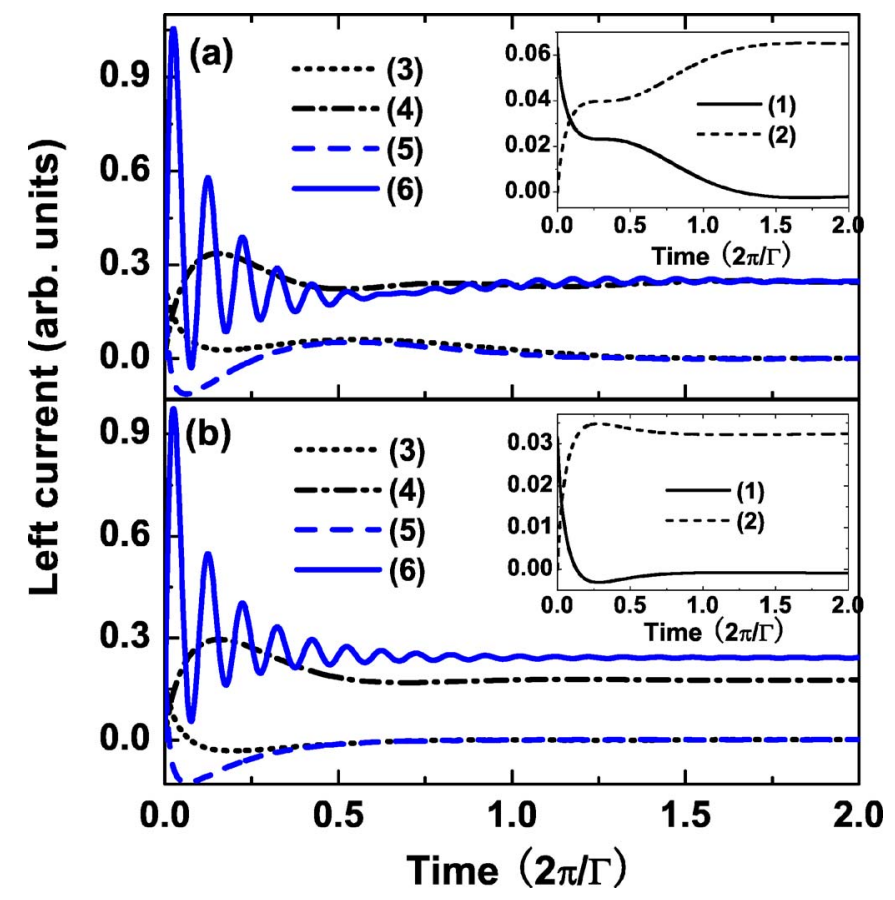

FIG. 3. (Color online) The currents $J_{L}(t)$ vs time $t$ for the N-QD-S system (a) and the N-QD-N system (b) with the different pulsed bias $V_{L}$. The main figure is for the case of $V_{L}=1$ and $V_{L}$ $=10$. The case of $V_{L}=0.1$ is plotted in the inset panel. The curves are labeled as (1) $J_{L}^{D}\left(V_{L}=0.1\right)$, (2) $J_{L}^{U}\left(V_{L}=0.1\right),(3) J_{L}^{D}\left(V_{L}=1.0\right)$, (4) $J_{L}^{U}\left(V_{L}=1.0\right)$, (5) $J_{L}^{D}\left(V_{L}=10\right)$, and (6) $J_{L}^{U}\left(V_{L}=10\right)$. The other parameters are the same as in Fig. 1.

pends on the coupling strength $\Gamma$. Let us explain why the character of $J_{L}(t)$ for the normal and hybrid systems are the same at large $V_{L}$ but very different at small $V_{L}$. Because at the large bias $V_{L}$, most of the incoming electrons have the large energy $\omega, T_{A} \ll 1$ from Eq. (17) and the Andreev reflection is weak, so the N-QD-S device and the N-QD-N device have the same turn-on/off time and relaxation time. But for the small bias $V_{L}$, the resonant Andreev reflection is dominant in the transport process in the hybrid system, so that the current $J_{L}(t=\infty)$ of the hybrid system is twice as that of the normal system, and their character $J_{L}(t)$ are also very different. So we will only discuss the small pulsed bias $V_{L}$ case further in the following.

Finally, we consider the case of asymmetric barriers (i.e., $\left.\delta \Gamma=\Gamma_{L}-\Gamma_{R} \neq 0\right)$ and in the small pulsed bias $V_{L}$. Because in the small $V_{L}$ the time-dependent current $J_{L}(t)$ for the upward and downward pulses are symmetric, we only study the upward pulse case. Figure 4 plots the current $J_{L}^{U}(t)$ versus time $t$ for the different asymmetric coupling strengths $\delta \Gamma$, and they have the following behaviors: (i) As $\delta \Gamma$ (i.e., $\Gamma_{L}$ ) increases, the current $J_{L}^{U}(t)$ rises faster, i.e., the turn-on time is shorter, because electrons with energy in the bias window can tunnel through the left barrier more easily with the larger $\Gamma_{L}$. This rising process of $J_{L}^{U}$ are nearly the same for the normal and hybrid systems. (ii) After the rise of $J_{L}^{U}$ [at $t$ $\simeq 0.2(2 \pi / \Gamma)]$, Andreev reflection begins to dominate and gives rise to different sequent relaxation processes for the normal and hybrid systems. At $\delta \Gamma<0, J_{L}^{U}(t)$ of the hybrid

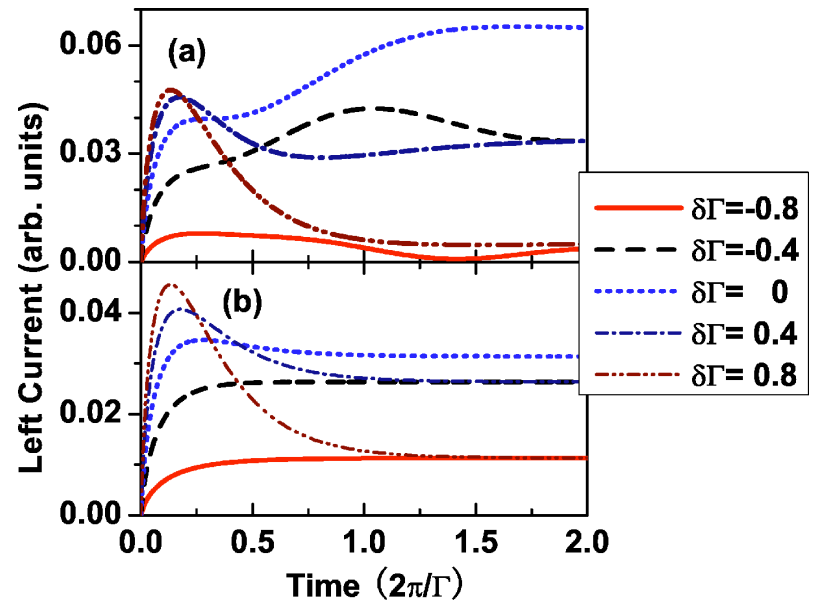

FIG. 4. (Color online) The current $J_{L}^{U}(t)$ vs the time $t$ in the small pulse bias $V_{L}=0.1$ with the different asymmetric coupling strength $\delta \Gamma$. (a) and (b) are for the N-QD-S system and the N-QD-N system, respectively. The other parameters are the same as those in Fig. 1.

system humps slightly in the relaxation process, which is obviously different from the normal system in which $J_{L}^{U}(t)$ is monotonically relaxed into the steady state. When $\delta \Gamma=0$, $J_{L}^{U}(t)$ of the hybrid system passes a step and increases again. The relaxation time for the hybrid system is much longer than that of the normal system when $\delta \Gamma \leqslant 0$. When $\delta \Gamma>0$, the relaxation processes of $J_{L}^{U}(t)$ are similar for the hybrid system and the normal system. These behaviors can be interpreted by combining the density of state (DOS) of the QD with the Andreev reflection possibility $T_{A}$. In fact, at $\delta \Gamma$ $>0$, the DOS of the QD in the hybrid system is similar to that of the normal system and $T_{A} \ll 1$, so that the two systems have similar turn-on/off and relaxation characteristics. On the other hand, when $\delta \Gamma=0$ or $\delta \Gamma<0$, the resonant or the near resonant Andreev reflection occurs, Andreev bound states appear in the QD, and the DOS of the QD is very different from the normal system. This makes the relaxation processes very different for the N-QD-S and N-QD-N systems. (iii) Although $J_{L}^{U}(t)$ for $\delta \Gamma=+a$ and $\delta \Gamma=-a$ ( $a$ is an arbitrary real number) experience different rising and relaxation processes, they have the same steady-state value at $t$ $=\infty$. In fact, in the steady-state case and at the small bias $V_{L}$ limit, the transmission possibility of the normal N-QD-N device is

$$
T(\omega)=\frac{\Gamma^{2}-\delta \Gamma^{2}}{4 \omega^{2}+\Gamma^{2}},
$$

and the Andreev reflection possibility of the hybrid N-QD-S device is ${ }^{21}$

$$
T_{A}(\omega)=\frac{\left(\Gamma^{2}-\delta \Gamma^{2}\right)^{2}}{4\left(4 \omega^{2}+\Gamma \delta \Gamma\right)^{2}+\left(\Gamma^{2}-\delta \Gamma^{2}\right)^{2}},
$$

with the current expressions $J_{L}=-2 q \int \frac{d \omega}{2 \pi}\left[f\left(\omega-V_{L}\right)\right.$ $-f(\omega)] T(\omega)$ and $J_{L}=-2 q \int \frac{d \omega}{2 \pi}\left[f\left(\omega-V_{L}\right)-f\left(\omega+V_{L}\right)\right] T_{A}(\omega)$, respectively. Here $T$ and $T_{A}$ are the same for $\pm \delta \Gamma$ when $\omega=0$; consequently, $J_{L}(t=\infty)$ are also the same for $\pm \delta \Gamma$. 


\section{CONCLUSIONS}

In summary, we have studied the dynamic response of current to the external upward or downward pulsed bias for the hybrid N-QD-S system. In the small bias $V_{L}$ limit, the turn-on/off time and the relaxation process for the upward and the downward pulse biases are symmetric. Comparing wtih the normal N-QD-N system, the Andreev reflection dominates the transport process. This makes the turn-on/off time much longer and the steady-state current almost doubled. For the asymmetric barriers, the transport properties of the hybrid N-QD-S system are nearly the same as those of the normal N-QD-N system when $\Gamma_{L}>\Gamma_{R}$. On the other hand, while $\Gamma_{L}<\Gamma_{R}$ the current humps in the relaxation process which reflects the properties of the superconductor. Beyond the linear bias regime, the rising process for upward bias and the falling process for the downward bias becomes more and more asymmetric with the increasing bias $V_{L}$. The turn-on time is faster than the turn-off time, and the current versus the time $t$ oscillates with the frequency $\hbar \Omega=V_{L}$.

\section{ACKNOWLEDGMENTS}

This work was supported by the Chinese Academy of Sciences and NSF-China under Grant Nos. 90303016, 10474125, and 10525418. J.W. is supported by RGC Grant No. HKU 7044/05P from the government SAR of Hong Kong and LuXin Energy Group.

\section{APPENDIX}

In this Appendix, we give the self-energy $\Sigma^{r,<}$ for coupling to the left normal and right superconductor leads. To consider the wideband limit, in which the hopping elements $t_{k, \alpha}$ is independent of the momentum $k$ and the density of state of the leads $\rho_{L / R}^{N}(E)$ is energy independent, the selfenergies $\Sigma_{L, \sigma}^{r,<}$ from the coupling to the left normal lead with the time-dependent bias potential $W_{L}(t)$ and in the Nambu representation are

$$
\Sigma_{L, \sigma}^{r}\left(t^{\prime}, t\right)=\sum_{k, L} t_{k, L}^{*} g_{k \sigma, L}^{r}\left(t^{\prime}, t\right) t_{k, L}=-\frac{i}{2} \Gamma_{L} \delta\left(t^{\prime}-t\right),
$$

$$
\begin{aligned}
\Sigma_{L, \uparrow}^{<}\left(t^{\prime}, t\right)= & \sum_{k, L} t_{k, L}^{*} g_{k \uparrow, L}^{<}\left(t^{\prime}, t\right) t_{k, L} \\
= & i \int \frac{d \omega}{2 \pi} f(\omega) \Gamma_{L} \\
& \times \exp \left[-i \omega\left(t^{\prime}-t\right)-i \int_{t}^{t^{\prime}} d t_{1} W_{L}\left(t_{1}\right)\right], \\
\Sigma_{L, \downarrow}^{<}\left(t^{\prime}, t\right)= & \sum_{k, L} t_{k, L}^{*} g_{k \downarrow, L}^{<}\left(t^{\prime}, t\right) t_{k, L} \\
= & i \int \frac{d \omega}{2 \pi}(1-f(\omega)) \Gamma_{L} \\
& \times \exp \left[i \omega\left(t^{\prime}-t\right)+i \int_{t}^{t^{\prime}} d t_{1} W_{L}\left(t_{1}\right)\right] \\
= & i \int \frac{d \omega}{2 \pi} f(\omega) \Gamma_{L}\left[-i \omega\left(t^{\prime}-t\right)+i \int_{t}^{t^{\prime}} d t_{1} W_{L}\left(t_{1}\right)\right] . \\
& \times \exp [
\end{aligned}
$$

Here $\Gamma_{L}=2 \pi\left|t_{k, L}\right|^{2} \rho_{L}^{N}, g_{k \sigma, L}^{r,<}\left(t^{\prime}, t\right)$ is the Green's function of the isolated left lead, and $f(\omega)$ is the Fermi distribution. Notice that the retarded self-energy $\Sigma_{L, \sigma}^{r}\left(t^{\prime}, t\right)$ is not affected by the time-dependent bias $W_{L}(t)$, so it is still a function of the time difference $t^{\prime}-t$. Since the time-dependent bias $W(t)$ is applied only on the left normal lead and $W_{R}(t)=0$, so the self-energies for coupling to the right superconductor lead are the same as those of the steady-state case and they can be written as ${ }^{7,21}$

$$
\begin{gathered}
\Sigma_{R}^{r}(\omega)=-i \frac{\Gamma_{R}}{2} \frac{\nu}{\sqrt{\omega^{2}-\Delta^{2}}}\left(\begin{array}{cc}
\omega & \Delta \\
\Delta & \omega
\end{array}\right), \\
\Sigma_{R}^{<}(\omega)=i \theta(\omega-\Delta) f_{R}(\omega) \frac{\Gamma_{R}}{2} \frac{1}{\sqrt{\omega^{2}-\Delta^{2}}}\left(\begin{array}{cc}
\omega & \Delta \\
\Delta & \omega
\end{array}\right),
\end{gathered}
$$

where $\Gamma_{R}=2 \pi\left|t_{k, R}\right|^{2} \rho_{R}^{N}, \Delta$ is the energy gap of the superconductor lead, and $\nu=1$ for $\omega>-\Delta$ and $\nu=-1$ otherwise.
*Electronic address: sunqf@aphy.iphy.ac.cn

${ }^{1}$ G. Garcia-Calderon, in The Physics of Low-Dimensional Semiconductor Structures, edited by Pl. Butcher, N. H. March, and M. P. Tosi (Plenum, New York, 1993), p. 267.

${ }^{2}$ A.-P. Jauho, N. S. Wingreen, and Y. Meir, Phys. Rev. B 50, 2008 (1994)

${ }^{3}$ L. P. Kouwenhoven, S. Jauhar, J. Orenstein, P. L. McEuen, Y. Nagamune, J. Motohisa, and H. Sakaki, Phys. Rev. Lett. 73, 3443 (1994); L. Y. Gorelik, Frank A. Maao, R. I. Shekhter, and M. Jonson, ibid. 78, 3169 (1997).

${ }^{4}$ Q.-F. Sun, J. Wang, and T.-H. Lin, Phys. Rev. B 58, 2008 (1998).
${ }^{5}$ T. H. Oosterkamp, T. Fujisawa, W. G. van der Wiel, K. Ishibashi, R. V. Hijman, S. Tatucha, and L. P. Kouwenhoven, Nature (London) 395, 873 (1998); H. Drexler, J. S. Scott, S. J. Allen, K. L. Campman, and A. C. Gossard, Appl. Phys. Lett. 67, 2816 (1995).

${ }^{6}$ T. K. Ng, Phys. Rev. Lett. 76, 487 (1996).

${ }^{7}$ Q.-F. Sun, J. Wang, and T.-H. Lin, Phys. Rev. B 59, 13126 (1999).

${ }^{8}$ P. J. Burke, IEEE Trans. Nanotechnol. 2, 55 (2003); S. Li, Z. Yu, S. F. Yen, W. C. Tang, and P. J. Burke, Nano Lett. 4, 753 (2004).

${ }^{9}$ M. Plihal, D. C. Langreth, and P. Nordlander, Phys. Rev. B 61, 
R13341 (2000)

${ }^{10}$ A. Schiller and S. Hershfield, Phys. Rev. B 62, R16271 (2000).

${ }^{11}$ N. S. Wingreen, A.-P. Jauho, and Y. Meir, Phys. Rev. B 48, 8487 (1993).

${ }^{12}$ J. Maciejko, J. Wang, and H. Guo, Phys. Rev. B 74, 085324 (2006).

${ }^{13}$ Y. Zhu, J. Maciejko, T. Ji, H. Guo, and J. Wang, Phys. Rev. B 71, 075317 (2005)

${ }^{14}$ C. J. Lambert and R. Raimondi, J. Phys.: Condens. Matter 10, 901 (1998)

${ }^{15}$ A. F. Andreev, Sov. Phys. JETP 19, 1228 (1964).

${ }^{16}$ I. O. Kulic, Sov. Phys. JETP 57, 1745 (1969); J. Bardeen and J. L. Johnson, Phys. Rev. B 5, 72 (1972); A. F. Morpurgo, B. J. van Wees, T. M. Klapwijk, and G. Borghs, Phys. Rev. Lett. 79, 4010 (1997).

${ }^{17}$ F. Sols and J. Ferrer, Phys. Rev. B 49, 15913 (1994); M. Hurd, S. Datta, and P. F. Bagwell, ibid. 56, 11232 (1997); S. Ishizaka, J. Sone, and T. Ando, ibid. 52, 8358 (1995); R. Bauernschmitt, J. Siewert, Yu. V. Nazarov, and A. A. Odintsov, ibid. 49, 4076 (1994); P. F. Bagwell, ibid. 46, 12573 (1992); A. MartínRodero, F. J. García-Vidal, and A. Levy Yeyati, Phys. Rev. Lett. 72, 554 (1994); M. Hurd and G. Wendin, Phys. Rev. B 51, 3754 (1995).

${ }^{18}$ A. W. Kleinsasser, R. E. Miller, W. H. Mallison, and G. B. Ar- nold, Phys. Rev. Lett. 72, 1738 (1994); N. van der Post, E. T. Peters, I. K. Yanson, and J. M. van Ruitenbeek, ibid. 73, 2611 (1994); Q.-F. Sun, H. Guo, and J. Wang, Phys. Rev. B 65 , 075315 (2002).

${ }^{19}$ Concerning the related works on the N-QD-S system with the interaction but in the absence of an ac signal, one can refer to the following references: R. Fazio and R. Raimondi, Phys. Rev. Lett. 80, 2913 (1998); K. Kang, Phys. Rev. B 58, 9641 (1998); A. A. Clerk, V. Ambegaokar, and S. Hershfield, ibid. 61, 3555 (2000); Q.-F. Sun, H. Guo, and T.-H. Lin, Phys. Rev. Lett. 87, 176601 (2001); J. C. Cuevas, A. L. Yeyati, and A. MartínRodero, Phys. Rev. B 63, 094515 (2001).

${ }^{20}$ J. C. Cuevas, A. Martin-Rodero, and A. L. Yeyati, Phys. Rev. B 54, 7366 (1996).

${ }^{21}$ Q.-F. Sun, J. Wang, and T.-H. Lin, Phys. Rev. B 59, 3831 (1999); Q.-F. Sun, B.-G. Wang, J. Wang, and T.-H. Lin, ibid. 61, 4754 (2000).

${ }^{22}$ P. G. de Gennes, Superconductivity of Metals and Alloys (Benjamin, New York, 1996).

${ }^{23}$ Y. Nambu, Phys. Rev. 117, 648 (1960).

${ }^{24}$ Y. Xing, B. Wang, Y. Wei, B. Wang, and J. Wang, Phys. Rev. B 70, 245324 (2004).

${ }^{25}$ To go beyond the wideband limit, one has to use the approach of Ref. 12. 